\title{
Simultaneous measurements of dynamical structure in the mesopause region with lidars and MU radar
}

\author{
K. Kobayashi ${ }^{1 *}$, T. Kitahara ${ }^{1}$, T. D. Kawahara ${ }^{1}$, Y. Saito ${ }^{1}$, A. Nomura ${ }^{1}$, T. Nakamura ${ }^{2}$, T. Tsuda $^{2}$, \\ M. $\mathrm{Abo}^{3}$, C. Nagasawa ${ }^{3}$, and M. Tsutsumi ${ }^{4}$ \\ ${ }^{1}$ Faculty of Engineering, Shinshu University, Nagano, Japan \\ ${ }^{2}$ Radio Atmospheric Science Center, Kyoto University, Kyoto, Japan \\ ${ }^{3}$ Department of Electronics and Information Engineering, Tokyo Metropolitan University, Tokyo, Japan \\ ${ }^{4}$ National Institute of Polar Research, Tokyo, Japan
}

(Received August 4, 1998; Revised March 5, 1999; Accepted March 5, 1999)

\begin{abstract}
In order to clarify the horizontal structure of the wavelike oscillation frequently observed in the night time sodium density profile with a small Gaussian half-width in the middle of the night and a broad distribution at dusk, simultaneous observations with two lidars at Shigaraki $\left(34.9^{\circ} \mathrm{N}, 136.1^{\circ} \mathrm{E}\right)$ and Hachioji $\left(35.6^{\circ} \mathrm{N}, 139.4^{\circ} \mathrm{E}\right)$ and the MU radar at Shigaraki has been carried out. In the campaign of 35 nights, simultaneous observation was successful in four nights. On December 27-28, 1995, a large scale wave motion was observed by two lidars and the MU radar with meteor observation mode. Phase velocities were almost the same at the two sites and there were only slight differences in phase between the two sites. The wave motion was inconsistent with the component of the tidal wave and similar with the results of hodograph analyses. It is possible that the wave observed by lidars on December 27-28, 1995 was a gravity wave. The results of analyses suggest the possibility of gravity waves which were observed at fixed local time.
\end{abstract}

\section{Introduction}

In recent years significant efforts have been made to study the dynamical structure in the mesosphere and lower thermosphere (MLT) with active remote sensing techniques, such as lidars, radars and rockets, and with other passive optical instruments, such as airglow imagers (Fritts and Rastogi, 1985; Gardner and Voelz, 1987; Tsuda et al., 1990; Nakamura et al., 1993; Collins et al., 1994; Hostetler and Gardner, 1994; Taylor et al., 1995; Namboothiri et al., 1996). The MLT is energetically an important transition region between the lower and upper atmosphere regions. Atmospheric waves, such as gravity waves, tides and planetary waves, propagating up from lower levels of the atmosphere play important roles in dynamical processes such as energy transport and momentum transport, and influence the global atmospheric circulation. Unfortunately, the MLT is one of the least understood regions, even with remote sensing techniques.

The mesospheric sodium layer formed by meteoric ablation is an excellent tracer of the atmospheric wave motion, which is confined to the region between 80 and $100 \mathrm{~km}$ with a peak near the mesopause at $90-95 \mathrm{~km}$. A sodium lidar is a powerful tool for measuring sodium density profiles and their perturbations by atmospheric wave motions. A narrowband sodium lidar is capable of providing temperature profiles by detecting the thermally Doppler broadened Na D2 resonance structure (Bills et al., 1991; She et al., 1991). Sodium lidar

*Now at TRDI, Japan Defense Agency, Tokyo, Japan.

Copy right(C) The Society of Geomagnetism and Earth, Planetary and Space Sciences (SGEPSS); The Seismological Society of Japan; The Volcanological Society of Japan; The Geodetic Society of Japan; The Japanese Society for Planetary Sciences. measurements have provided useful information for clarifying the dynamical and chemical processes in the mesopause region since the early 1970s. Although the sodium layer has been mostly measured with high temporal and vertical resolution by ground-based lidars, except for air-borne lidar measurements by Kwon et al. (1990), lidars have not provided useful information about the horizontal structure of the atmospheric waves as well as the sodium layer.

We had also observed the mesospheric sodium layer for five years at Nagano city using the sodium lidar. In those results, sodium density profiles had sharp shapes in the middle of the night and broad shapes at dusk and dawn in winter season. It is caused by the wave motions which existed on the fixed local time. It has been deduced that the wave motions were tidal waves because they appeared at fixed local time. However, we could not identify clearly whether they are global wave motions such as atmospheric tides or local wave motions such as gravity waves because a lidar has only one-dimensional information of the vertical profile at one location. In order to identify the wave motions whether they are tidal waves or gravity waves, the simultaneous measurements have been carried out using two separately located sodium lidars and the MU radar. The simultaneous measurements have two aims. First is to use the sodium lidar and the MU radar observation at Shigaraki $\left(34.9^{\circ} \mathrm{N}, 136.1^{\circ} \mathrm{E}\right)$ in order to compare the results by observing the same waves with different instruments. Second is to use the sodium lidar observations at two sites, Shigaraki $\left(34.9^{\circ} \mathrm{N}, 136.1^{\circ} \mathrm{E}\right)$ near Kyoto and Hachioji in Tokyo $\left(35.6^{\circ} \mathrm{N}, 139.4^{\circ} \mathrm{E}\right)$ in order to study the horizontal structure of atmospheric wave motion. Such measurements with these three instruments have never 
been reported in anywhere in the world.

The sodium lidar of Shinshu University was transferred from Nagano to the MU radar site in fall, 1995, since then, we have made lidar measurements simultaneously. Up to now, during the campaign of 35 nights, we succeeded in simultaneous measurements only nine nights. In this paper, we discuss the vertical and horizontal wave motion on the basis of the data obtained on December 27-28, 1995.

\section{Sodium Lidars and MU Radar}

Simultaneous measurements have been carried out at Shigaraki and Hachioji. These two locations are illustrated in Fig. 1. The meteor observation by MU radar has been carried out by Kyoto University at Shigaraki and the sodium lidar measurements have been made by Shinshu University at Shigaraki and Tokyo Metropolitan University at Hachioji. These two sites are separated by a horizontal distance of 310 $\mathrm{km}$ and the baseline between them makes an angle of $14.5^{\circ}$ with the zonal direction. Weather is different at the both sites and it is seldom clear at the same time at both sites. The days of the simultaneous measurements are listed in Table 1. Up to now, during the campaign of 35 nights, we succeeded in simultaneous measurements on only nine nights. From the nine nights of simultaneous observations, we have only four days of data with high SNR and long overlapped periods.

The main system parameters of two sodium lidars and MU radar meteor observation used in the cooperative measurements are summarized in Table 2. For the sodium lidar of Shinshu University installed at Shigaraki, the transmitter consists of a coaxial flashlamp pumped dye laser, in which the spectral narrowing and tuning to the sodium $\mathrm{D}_{2}$ line (589 $\mathrm{nm}$ ) are made by three prisms and two temperature controlled

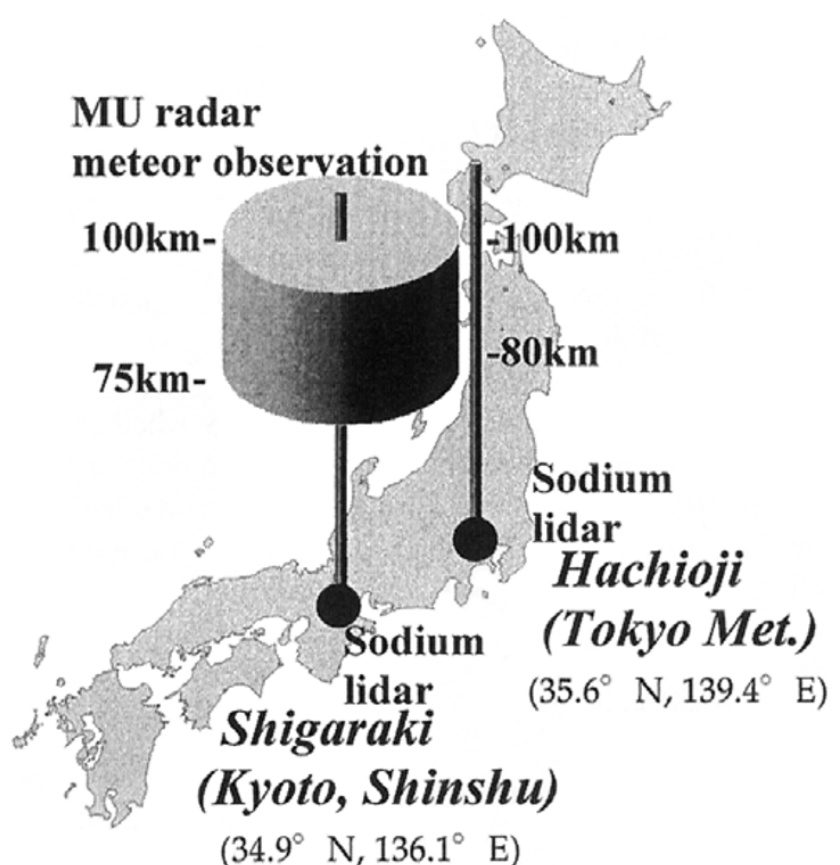

$300 \mathrm{~km}$
Fabry-Perot etalons inside the cavity. Laser output energy is $80-110 \mathrm{~mJ} /$ pulse with a repetition rate of $0.2 \mathrm{~Hz}$ and a linewidth of $3 \mathrm{pm}$. Scattered photons from each laser shot are collected by a Schmidt-Cassegrain telescope of $355 \mathrm{~mm}$ in diameter, passed through an interference filter, and detected by a photomultiplier tube (PMT). The amplified and discriminated photon signals are integrated over 100 laser shots by a range-gated photocounting system and processed by a personal computer. This system can provide sodium density profile every 10 minutes with a height resolution of $750 \mathrm{~m}$.

The sodium lidar of Tokyo Metropolitan University at Hachioji consists of a tunable dye laser pumped by a frequency doubled Nd:YAG laser, Hextek-Cassegrain telescope of $600 \mathrm{~mm}$ in diameter. Other components, such as a detector and a data processing system are almost same as the abovementioned system. Laser output energy tuned to the sodium resonance line with the linewidth of $2 \mathrm{pm}$ is $100 \mathrm{~mJ} /$ pulse with a repetition rate of $10 \mathrm{~Hz}$. This system can provide sodium density profiles every 4 minutes by integrating 2000 laser shots with a height resolution of $100 \mathrm{~m}$.

The MU radar of Kyoto University at Shigaraki is a monostatic pulse Doppler radar system with a frequency of 46.5 MHz (Nakamura et al., 1997). With the meteor echo observation mode, vertical wind structure of zonal and meridional components can be determined. At the same time, the MU radar meteor mode can observe ambipolar diffusion coefficients from the decay time constant of echo power and further derive temperature fluctuations in time (Tsutsumi et al., 1994). By analyzing the data obtained by the MU radar, we can obtain gravity wave parameters such as propagation direction and wave periods. The whole array with $1 \mathrm{MW}$ power and the interferometer using 4 Yagi antennas are used as the transmitter and the receiver, respectively. The radar can provide the vertical structure of wind and temperature every 30 minutes with a height resolution of $1 \mathrm{~km}$.

\section{Measurements and Comparison}

Simultaneous measurement with two lidars and the MU radar succeeded on December 27-28, 1995. Time-height variations of sodium density profiles at Shigaraki and Hachioji are shown in Fig. 2. Each profile is normalized by its column abundance. This normalization process of a vertical profile makes it difficult to detect wavelike variations with a long vertical wavelength, because the process eliminates long vertical wavelength components. Profiles are low-pass filtered vertically with a cutoff at $8 \mathrm{~km}$. Lack of data at Shigaraki indicates the discontinuations of measurements because of the cloudy sky and exchanges of the dye.

In these figures sodium density profiles had small Gaussian half-width in the middle of the night and had broad distributions at dusk and dawn in both sites. In order to extract the wave motion, time variations of sodium density profiles were converted to atmospheric density perturbations using the equation (Chiu and Ching, 1978; Gardner and Shelton, 1985).

$$
r_{\mathrm{s}}(z, t)=\frac{-1}{(\gamma-1)}\left[1-\frac{\gamma H\left(z-z_{0}\right)}{\sigma_{0}^{2}}\right] r_{\mathrm{a}}(z, t),
$$


Table 1. Simultaneous measurements.

\begin{tabular}{|c|c|c|c|}
\hline Day & Shinshu Univ. & Tokyo Met. Univ. & Kyoto Univ. \\
\hline Nov. 16, 1995 & 0 & & \\
\hline Nov. 17 & & 0 & $\bullet$ \\
\hline Nov. 18 & 0 & $\bullet$ & ○ \\
\hline Nov. 19 & & $\bullet$ & $\bullet$ \\
\hline Nov. 22 & 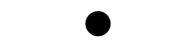 & & \\
\hline \multicolumn{4}{|l|}{ Nov. 25} \\
\hline Dec. 21, 1995 & & $\bullet$ & $\bullet$ \\
\hline Dec. 22 & $\bullet$ & & $\bullet$ \\
\hline Dec. 23 & $\bullet$ & & $\bullet$ \\
\hline Dec. 24 & & & 0 \\
\hline Dec. 27 & 0 & $\bullet$ & $\bullet$ \\
\hline Aug. 9, 1996 & ○ & & $\bullet$ \\
\hline Aug. 10 & $\bullet$ & $\triangle$ & $\bullet$ \\
\hline Aug. 11 & $\bullet$ & & $\bullet$ \\
\hline Aug. 12 & & $\triangle$ & $\bullet$ \\
\hline Aug. 13 & & & $\bullet$ \\
\hline Aug. 14 & & & $\bullet$ \\
\hline Oct. 14,1996 & 0 & & $\bullet$ \\
\hline Oct. 15 & 0 & $\triangle$ & $\bullet$ \\
\hline Oct. 16 & $\bullet$ & $\triangle$ & $\bullet$ \\
\hline Nov. 13, 1996 & $\bullet$ & $\bullet$ & $\bullet$ \\
\hline Nov. 14 & $\bullet$ & $\bullet$ & $\bullet$ \\
\hline Nov. 15 & $\bullet$ & $\bullet$ & $\bullet$ \\
\hline Nov. 16 & - & $\bullet$ & $\bullet$ \\
\hline Nov. 17 & & - & $\bullet$ \\
\hline Jan. 10, 1997 & & $\bullet$ & $\bullet$ \\
\hline Jan. 11 & & $\bullet$ & $\bullet$ \\
\hline Jan. 12 & - & $\bullet$ & $\bullet$ \\
\hline Jan. 13 & $\bullet$ & $\bullet$ & \\
\hline Jan. 14 & & & $\bullet$ \\
\hline Jan. 15 & $\bullet$ & $\bullet$ & $\bullet$ \\
\hline Jan. 16 & $\bullet$ & & $\bullet$ \\
\hline Jan. 17 & $\bullet$ & $\bullet$ & $\bullet$ \\
\hline Jan. 18 & $\triangle$ & $\bullet$ & $\bullet$ \\
\hline Jan. 19 & 0 & & 0 \\
\hline
\end{tabular}

$$
r_{\mathrm{s}}(z, t)=\rho_{\mathrm{s}}^{\prime} / \rho_{\mathrm{s} 0}, \quad r_{\mathrm{a}}(z, t)=\rho_{\mathrm{a}}^{\prime} / \rho_{\mathrm{a} 0},
$$

$\rho_{\mathrm{s}}^{\prime} / \rho_{\mathrm{s} 0}:$ Relative sodium density fluctuations, $\rho_{\mathrm{a}}^{\prime} / \rho_{\mathrm{a} 0}:$ Relative atmospheric density fluctuations,

$\gamma:$ Ratio of the specific heats $(\sim 1.4)$,

$H:$ Atmospheric scale height $(\sim 6 \mathrm{~km})$, $z_{0}:$ Centroid height of the unperturbed sodium layer $(\sim 92 \mathrm{~km})$,

$\sigma_{0}:$ RMS thickness of the unperturbed sodium layer $(\sim 4.3 \mathrm{~km})$.

The prime in $\rho_{\mathrm{a}}^{\prime}$ and $\rho_{\mathrm{s}}^{\prime}$ denotes the perturbation quantity, 
Table 2. System parameters of two sodium lidars and the MU radar.

\begin{tabular}{lll} 
Sodium lidar & & \\
\hline University & Shinshu & Tokyo Metropolitan \\
\hline Location & Shigaraki & Hachioji \\
& $\left(34.9^{\circ} \mathrm{N}, 136.1^{\circ} \mathrm{E}\right)$ & $\left(35.6^{\circ} \mathrm{N}, 139.4^{\circ} \mathrm{E}\right)$ \\
\hline Laser & Flashlamp-pumped & Nd:YAG SHG-pumped \\
& dye laser & dye laser \\
Pulse rate & $0.2 \mathrm{~Hz}$ & $10 \mathrm{~Hz}$ \\
Output power & $20 \mathrm{~mW}$ & $1 \mathrm{~W}$ \\
Wavelength & $589 \mathrm{~nm}$ & $589 \mathrm{~nm}$ \\
Linewidth & $3 \mathrm{pm}$ & $2 \mathrm{pm}$ \\
& $10 \mathrm{pm}($ after Aug. 1996) & \\
\hline Telescope aperture & $355 \mathrm{~mm}$ & $600 \mathrm{~mm}$ \\
Optical bandwidth & $1.0 \mathrm{~nm}$ & $3.5 \mathrm{~nm}$ \\
Field of view & $2 \mathrm{mrad}$ & $0.7 \mathrm{mrad}$ \\
\hline P.A. Product & $2.0 \times 10^{-3} \mathrm{Wm}^{2}$ & $2.8 \times 10^{-1} \mathrm{Wm}^{2}$ \\
Detection & \multicolumn{2}{c}{ Range-gated photocounts } \\
Height resolution & $750 \mathrm{~m}$ & $100 \mathrm{~m}$ \\
Time resolution & $10 \mathrm{~min}$. & $4 \mathrm{~min}$. \\
\hline
\end{tabular}

\begin{tabular}{ll} 
MU radar Meteor observation & \\
\hline University & Kyoto \\
\hline Location & Shigaraki $\left(34.9^{\circ} \mathrm{N}, 136.1^{\circ} \mathrm{E}\right)$ \\
\hline Operational Frequency & $46.5 \mathrm{MHz}$ \\
Transmission & Whole array-1 MW \\
Reception & Interferometer using 4 Yagi antennas \\
Time resolution & 30 min. \\
Height resolution & $1 \mathrm{~km}$ \\
\hline
\end{tabular}

while the zero in $\rho_{\mathrm{a} 0}$ and $\rho_{\mathrm{s} 0}$ indicates the associated mean quantity. In the analyses, associated mean sodium density was estimated from the profile averaged during each night. Centroid height was calculated from the averaged profile.

Atmospheric density perturbations $\rho_{\mathrm{a}}^{\prime} / \rho_{\mathrm{a} 0}$ at Shigaraki and Hachioji are shown in Fig. 3. Contours are filtered vertically with a cutoff of $8 \mathrm{~km}$ and perturbations are shown in order to clarify wave motions. White and dark gray regions indicate positive and negative perturbations respectively. The black region indicates no data. It is difficult to calculate the perturbations at peak of sodium layer in using Eq. (1). The perturbations exceeding $\pm 40 \%$ in the region of the sodium layer peak are indicated as gray color. In these calculations, $\rho_{\mathrm{a}}^{\prime} / \rho_{\mathrm{a} 0}$ varied from $6 \%$ to $-9 \%$ in the region between $82 \mathrm{~km}$ and $92 \mathrm{~km}$. The lower line in the figure was obtained by applying a least square fit to the zero points of $\rho_{\mathrm{a}}^{\prime} / \rho_{\mathrm{a} 0}$ during the period from 2330 (LT) to 0500 (LT). The upper line was obtained from 0330 (LT) to 0530 (LT). Atmospheric waves are shown during the period from 0000(LT) to 0600(LT). By calculating the average of the slopes of the upper and lower line, the wave had a vertical phase velocity of $-2.3 \mathrm{~km} / \mathrm{h}$ at Shigaraki. $\rho_{\mathrm{a}}^{\prime} / \rho_{\mathrm{a} 0}$ also varied from $6 \%$ to $-9 \%$ in the region between $82 \mathrm{~km}$ and $92 \mathrm{~km}$ at Hachioji. The lower line in the figure was obtained from a least square fit to the zero points of $\rho_{\mathrm{a}}^{\prime} / \rho_{\mathrm{a} 0}$ during the period from 0100 (LT) to 0500 (LT). The upper line was obtained from 0000 (LT) to 0430 (LT). By calculating the average of the slopes of the upper and lower line, the wave had a vertical phase velocity of $-1.9 \mathrm{~km} / \mathrm{h}$. Comparing $-2.3 \mathrm{~km} / \mathrm{h}$ with $-1.9 \mathrm{~km} / \mathrm{h}$, we found almost the same phase velocity between two sites. Calculating the time difference between two lines in the height range from $83 \mathrm{~km}$ to $88 \mathrm{~km}$ was 25 minutes. The wave period of about 8 hours is estimated from the difference between the upper line and the lower line in both figures. Time-height variations of northward and eastward wind velocities from MU radar on December 27-28, 1995 are shown in Fig. 4. Clear wave 

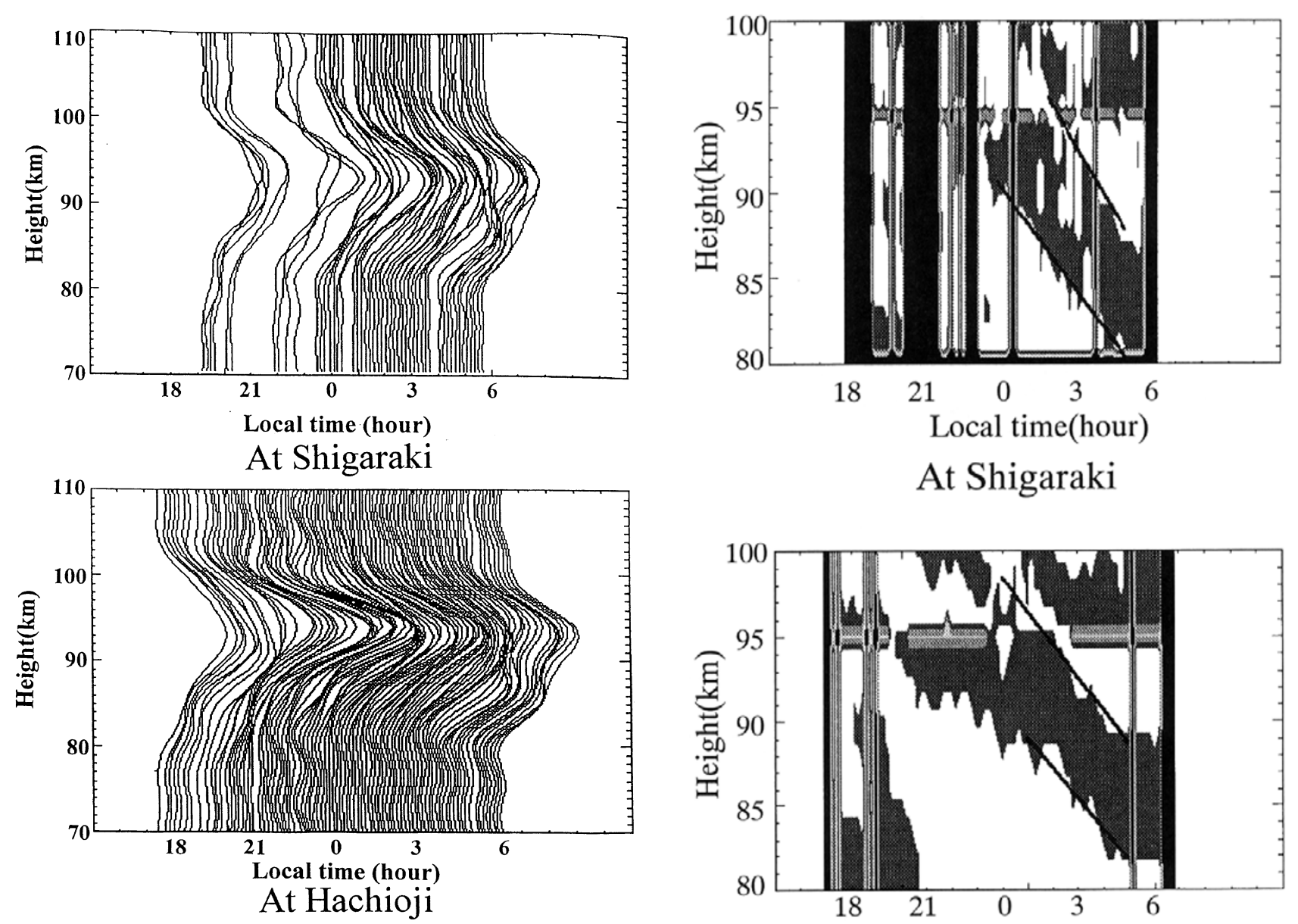

Fig. 2. Time-height variations of normalized sodium density profiles at Shigaraki and Hachioji obtained on Dec. 27-28, 1995.

motions were observed after midnight when waves were observed by lidars at Shigaraki and Hachioji.

Next, we compare atmospheric density perturbations with diffusion coefficient perturbations. Diffusion coefficient perturbations obtained from the MU radar meteor observation are shown in Fig. 5. The perturbations were low-pass filtered vertically with a cutoff at $8 \mathrm{~km}$. Diffusion coefficient perturbations are in proportion to temperature perturbations under a Boussinesq approximation. Comparing the atmospheric density perturbation at Shigaraki (Fig. 3) with diffusion coefficient perturbations (Fig. 5), there are regions where the phases are similar. Below $90 \mathrm{~km}$ between 0000 (LT) and 0400 (LT), the positive region of $\rho_{\mathrm{a}}^{\prime} / \rho_{\mathrm{a} 0}$ almost coincides with the region under the value of 1.0 on the diffusion coefficient perturbations. The negative region of $\rho_{\mathrm{a}}^{\prime} / \rho_{\mathrm{a} 0}$ almost coincides with the region over the value of 1.0 on the diffusion coefficient perturbations. And the apparent downward phase velocity is similar. But there are regions where phases are not consistent, for example, the region above $90 \mathrm{~km}$ between 0000 (LT) and 0400 (LT). Two reasons are suggested for the disagreement. First, the variations with a long vertical wavelength are filtered out in the results, so the lidar data showed a shorter vertical wavelength. Second, the atmospheric density perturbations are equated to the temperature fluctuation due to gravity waves applying the Boussinesq ap-

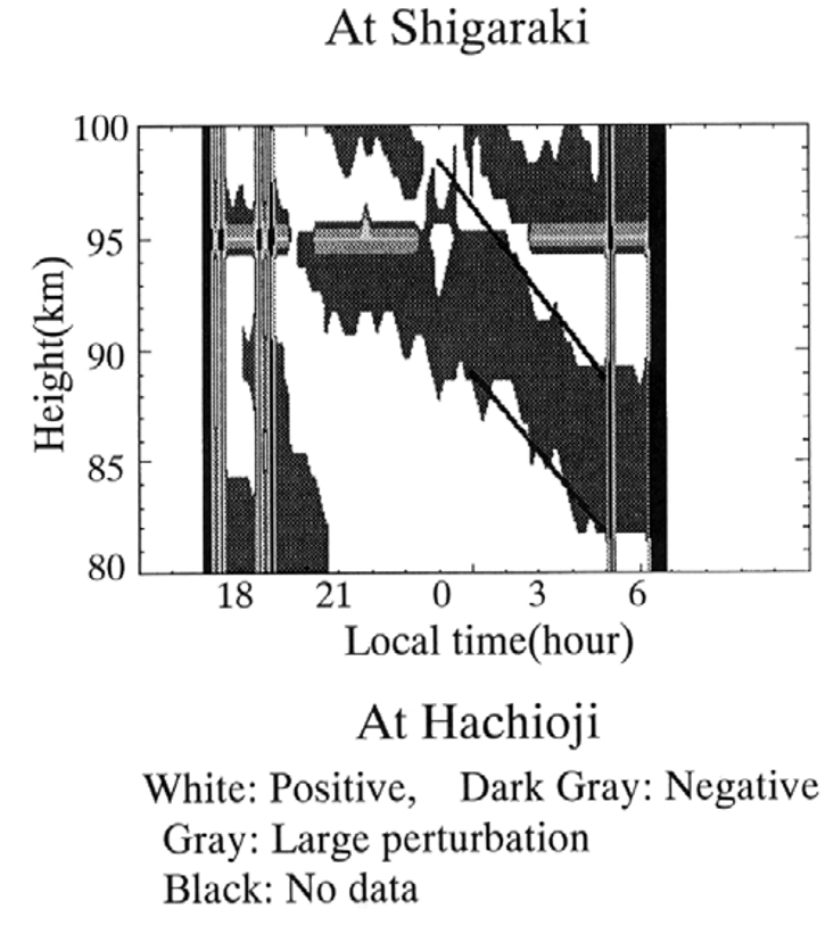

Fig. 3. Atmospheric density perturbations (filtered vertically with a cutoff of $8 \mathrm{~km}$ ) at Shigaraki and Hachioji (Dec. 27-28, 1995).

proximation. But the relation is not true for the global waves such as tidal waves.

In order to investigate the wave obtained from lidar data analyses, the atmospheric density perturbations were compared with the time variations of meridional and zonal wind velocities. In the meridional wind component, a similar wave motion appeared, but downward phase velocities were slightly different. Wave motions resembling tidal waves were shown in the meridional wind velocity variations between December 21-28, 1995 from the MU radar data. Wind velocity components consist of various waves, gravity waves, tidal waves, and so on. Therefore, we extracted the components of tidal waves from the wind velocity components. The tidal wave components and mean wind velocities calculated from the data between December 22-28, 1995 by a least square fitting of sinusoidal waves to the MU radar wind data are shown in Fig. 6. The data of 109 hours were used in tidal fitting. The lower eight figures show the diurnal tide 
Table 3. Gravity wave parameters obtained from the hodograph analyses of height profiles of wind velocity observed with the MU radar on Dec. 27-28, 1995.

\begin{tabular}{cccccc}
\hline Local time & $\mathrm{T}(\mathrm{hr})$. & P.D. (deg.) & $\lambda_{\mathrm{z}}(\mathrm{km})$ & $\lambda_{\mathrm{h}}(\mathrm{km})$ & $\mathrm{C}_{\mathrm{h}}(\mathrm{m} / \mathrm{s})$ \\
\hline $0: 30$ & 8.7 & -1.6 & 10 & 1184 & 37.8 \\
$1: 00$ & 9.7 & -1.7 & 10 & 1354 & 38.9 \\
$1: 30$ & 10.2 & 0.7 & 12 & 1734 & 47.2 \\
$2: 00$ & 12.4 & -5.7 & 14 & 2664 & 60.0 \\
$2: 30$ & 10.3 & -3.1 & 13 & 1184 & 51.3 \\
$3: 00$ & 7.5 & 1.1 & 12 & 1193 & 44.2 \\
\hline Average & 9.8 & -1.7 & 12 & 1552 & 46.6 \\
\hline
\end{tabular}

T: Wave perild (hours), P.D.: Propagation Direction (Northward $0^{\circ}$, Eastward $\left.90^{\circ}\right), \lambda_{\mathrm{z}}(\mathrm{km})$ : Vertical wavelength, $\lambda_{h}(\mathrm{~km})$ : Horizontal wavelength, $C_{h}(\mathrm{~m} / \mathrm{s})$ : Horizontal propagation velocity.
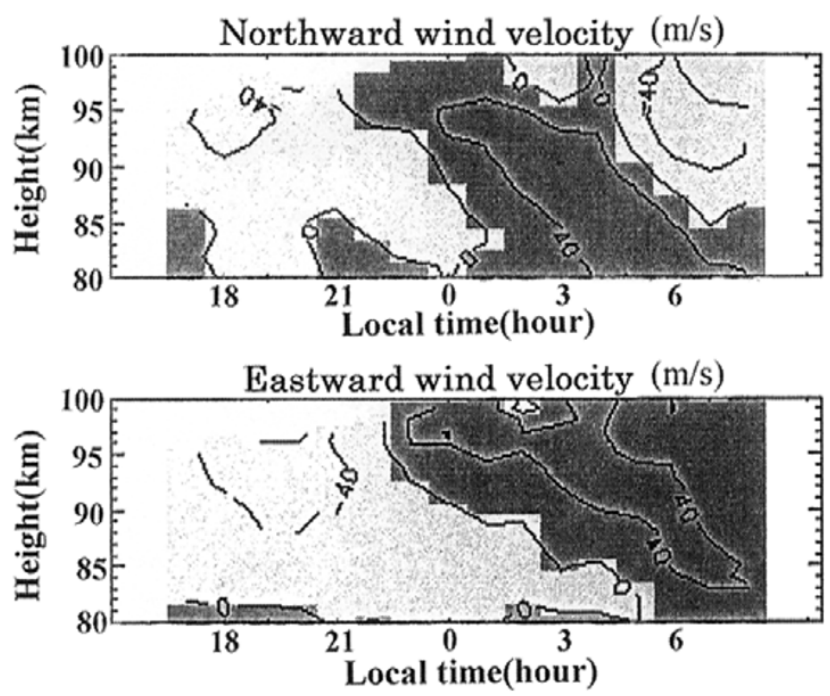

Fig. 4. Time-height variations of northward and eastward wind velocities on December 27-28, 1995 obtained from the MU meteor observation.

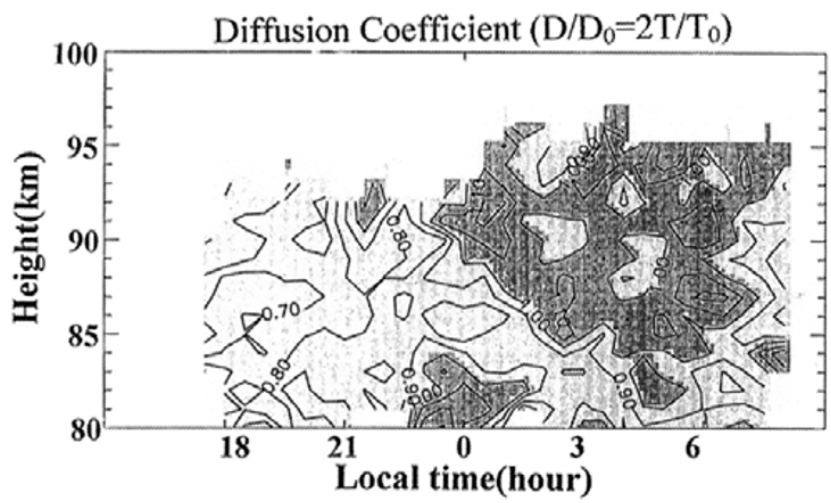

Fig. 5. Time variations of Diffusion coefficient perturbations on December 27-28, 1995 obtained from the MU meteor observation. components and semidiurnal tide components. On each four figures, upper figures show the zonal components, and lower figures show the meridional components. From phase-height variations, the component of the tidal wave had the vertical wavelength of $57 \sim 76 \mathrm{~km}$ and the semidiurnal tide had the vertical wavelength of $35 \sim 38 \mathrm{~km}$. However these wavelengths were much longer than lidars could identify. Those tidal components are restructured in Fig. 7. The slope of downward phase is about $-3.7 \mathrm{~km} / \mathrm{h}$ from MU radar data and it is different from the results of sodium lidars. So, it is inferred that the wave motion obtained from the lidars was not tidal.

By hypothesizing that the wave is a gravity wave, the gravity wave parameters are obtained using the hodograph analyses (Tsuda et al., 1990). Horizontal structure of a gravity wave is calculated by Eqs. (2) and (3). A fundamental dispersion relation between the intrinsic wave frequency, $\omega$, and vertical and total horizontal wavenumber, $m$ and $k$, can be written as

$$
m^{2}=\frac{N^{2}-\omega^{2}}{\omega^{2}-f^{2}} k^{2}
$$

where $N$ and $f$ are Brunt-Väisälä and inertial frequency, respectively, and $-1 / 4 H^{2}$ can be neglected (Hines, 1960; Gossard and Hooke, 1975). The value of the inertial frequency $f$ was used $1.33 \times 10^{-5} \mathrm{~Hz}$ at the latitude of $35^{\circ}$ at Shigaraki, corresponding to a period of 20.9 hours. We assumed the value of $N$ as $3.33 \times 10^{-3} \mathrm{~Hz}$ (Namboothiri et al., 1996). The intrinsic frequency of the wave can be estimated with the gravity wave polarization relation

$$
\frac{v^{\prime}}{u^{\prime}}=-i \frac{f}{\omega}
$$

where $u^{\prime}$ and $v^{\prime}$ are the horizontal perturbation velocities parallel and perpendicular, respectively, to the direction of horizontal propagation (Namboothiri et al., 1996).

The data with the wind velocities of tidal wave components were subtracted from the original wind velocities and were used in the hodograph analyses. Table 3 summarizes the value of the gravity wave parameters during the period from 0030 (LT) to 0300 (LT). The average value of the wave period, 9.8 hours was close to the value obtained from 

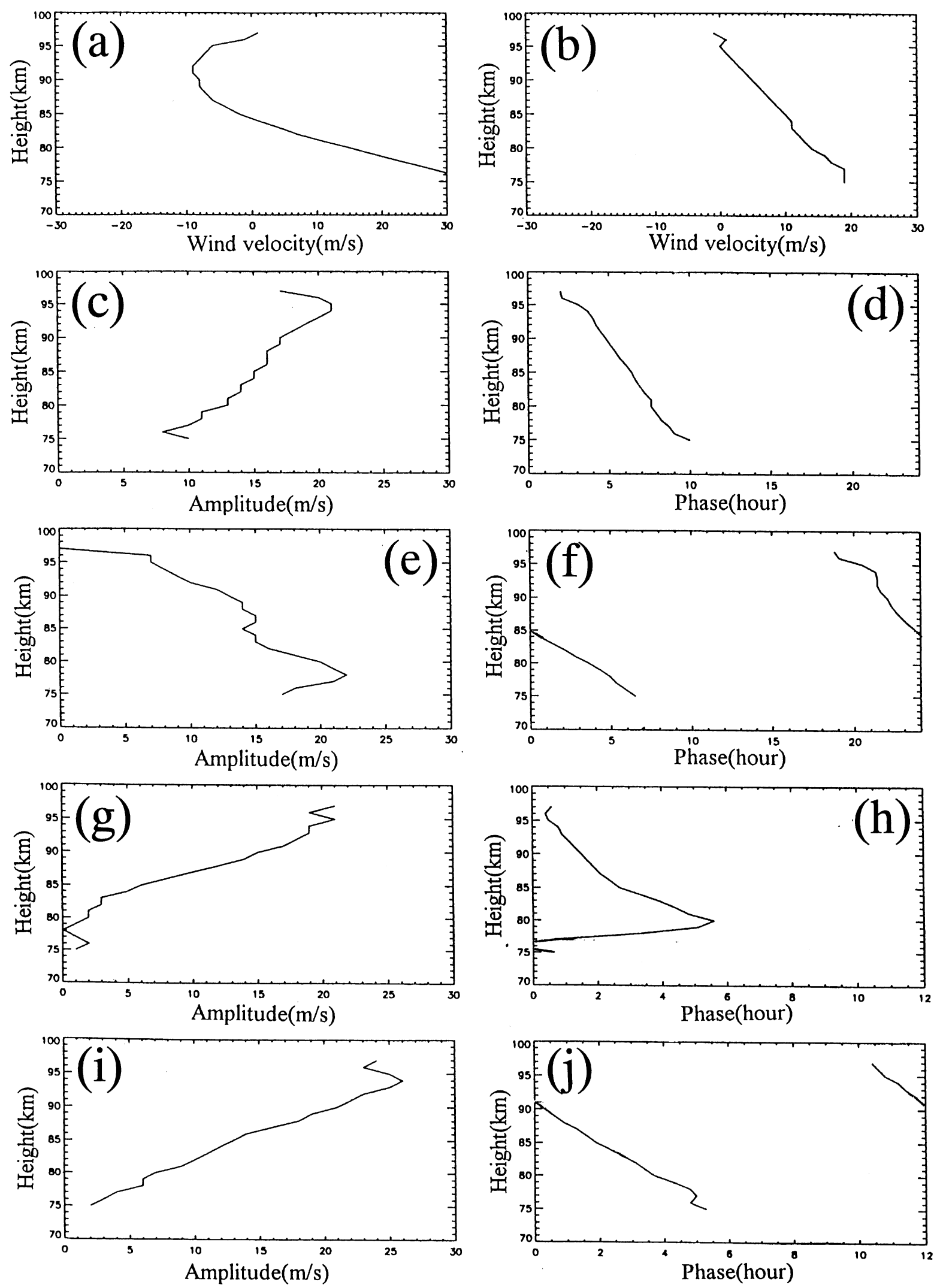

Fig. 6. Tidal wave components and mean wind velocities obtained from December 22, 1995 until December 28, 1995. (a) Zonal (b) Meridional (c) Amplitude of diurnal component of zonal wind (d) Phase of diurnal component of zonal wind (e) Amplitude of diurnal component of meridional wind (f) Phase of diurnal component of meridional wind (g) Amplitude of semidiurnal component of zonal wind (h) Phase of semidiurnal component of zonal wind (i) Amplitude of semidiurnal component of meridional wind (j) Phase of semidiurnal component of meridional wind. 

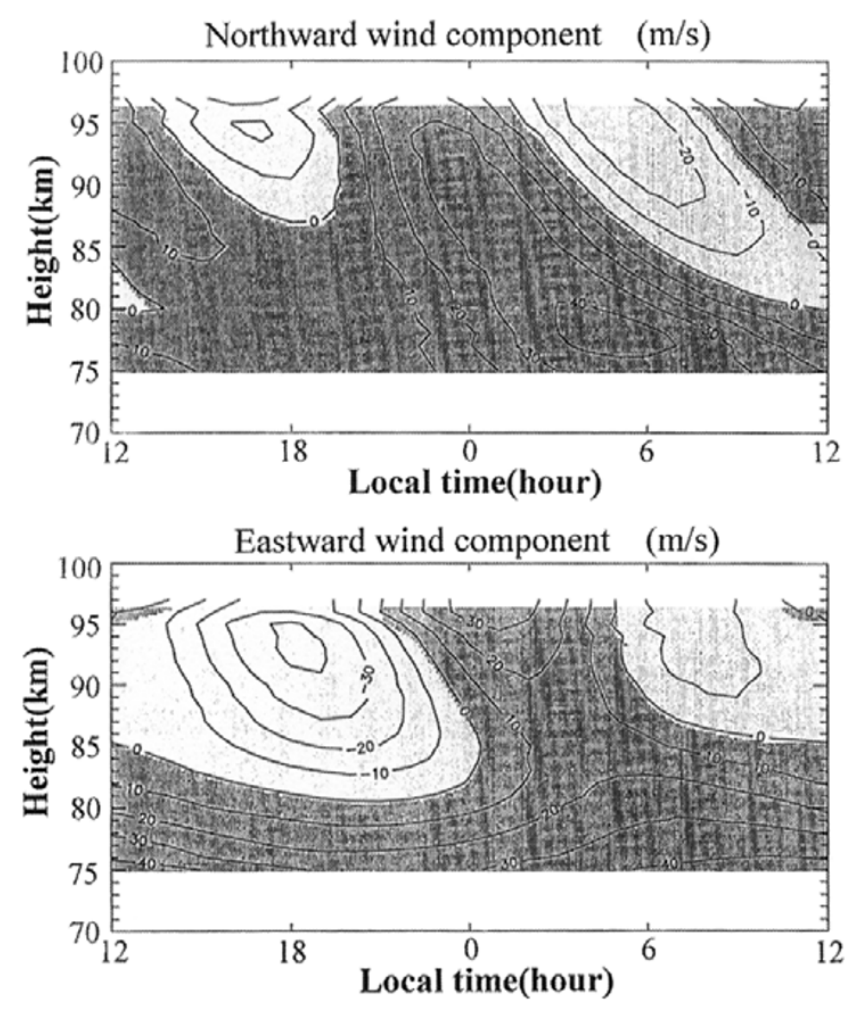

Fig. 7. Wind velocity components of the restructured tidal components.

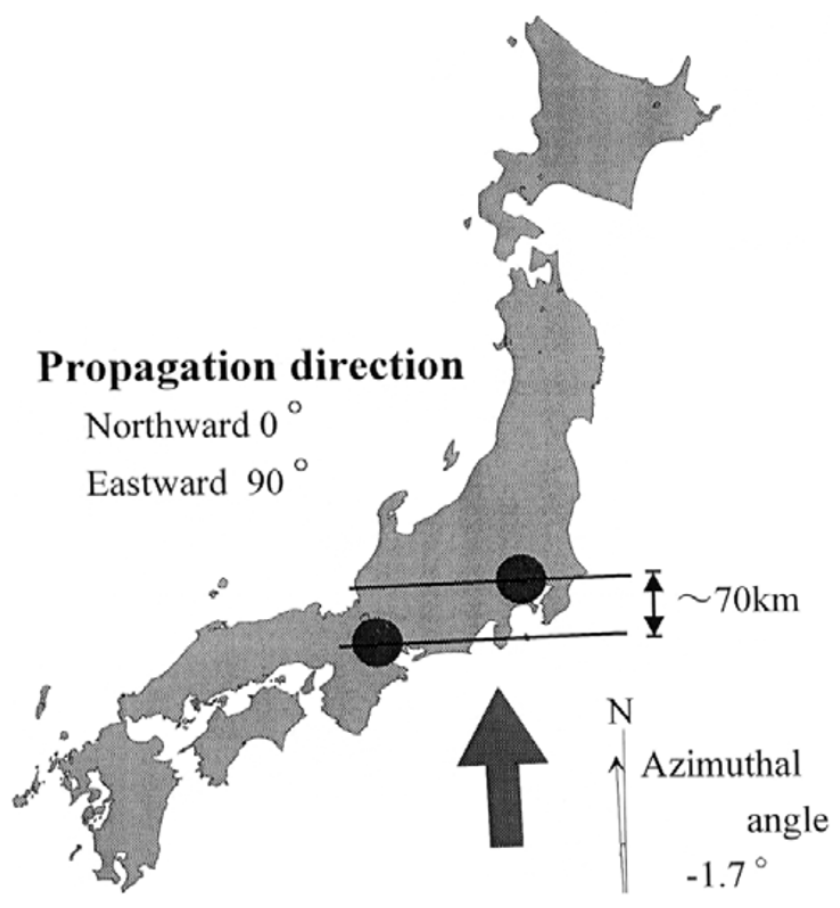

Fig. 8. The horizontal propagation of the gravity wave on December 28, 1995. The arrow schematically indicates the horizontal propagation direction -1.7 degree of the gravity wave.

lidars. The propagation direction of the gravity wave was estimated -1.7 degree, in which the direction from south to north was defined as 0 degree and the direction from west to east was defined as 90 degree. The horizontal propagation of the gravity wave is shown in Fig. 8. The arrow schematically indicates the horizontal propagation direction -1.7 degree of the gravity wave. If the gravity wave propagates toward the direction $(-1.7$ degree $)$, the distance from Shigaraki to Hachioji along the propagating direction is approximately $70 \mathrm{~km}$. From the average horizontal perturbation velocity and the mean wind velocity obtained from Fig. 6, the arrival time lag of the gravity wave between Shigaraki and Hachioji was estimated about 20 minutes. This time lag agrees well with 25 minutes obtained from lidars. Consequently, it is possible that the wave obtained from the results of lidars was a gravity wave. Assuming that the wave obtained from lidars was a gravity wave, disagreement regions comparing the atmospheric density perturbation (Fig. 3) with diffusion coefficient perturbations (Fig. 5) were caused by the first reason as mentioned above, the MU radar data included waves from tidal waves with long vertical wavelength to gravity waves with short vertical wavelength, whereas the lidar data didn't include waves with long vertical wavelength because of the normalization.

\section{Conclusion}

We have observed mesospheric sodium layers and meteor echo in the mesopause region simultaneously using two sodium lidars at Shigaraki and Hachioji and the MU radar at Shigaraki since November, 1995. The simultaneous measurements were carried out in order to identify wave motions whether the wave motions which were observed at a fixed local time are global wave motions such as atmospheric tides or mesoscale wave motions such as gravity waves. Simultaneous measurement succeeded on December 27-28, 1995 with high SNR. A large scale atmospheric wave was observed by lidars during the period from 0000 (LT) to 0600 (LT), which show similar phase velocity at both sites. There were only slight differences in phase between two sites. The wave motion was inconsistent with the component of the tidal wave. The wave motion was nearly consistent with the results of hodograph analyses. It is possible that the wave obtained from lidars on December 27-28, 1995 was a gravity wave. The results of analyses suggest the possibility that gravity waves caused the wave motion which was observed at fixed local time.

We intend to analyze another data set and continue this campaign and obtain many interesting results.

\section{References}

Bills, R. E., C. S. Gardner, and C. Y. She, Narrowband lidar technique for sodium temperature and Doppler wind observations of the upper atmosphere, Opt. Eng., 30, 13-21, 1991.

Chiu, Y. T. and B. K. Ching, The response of atmospheric and lower ionospheric layer structures to gravity waves, Geophys. Res. Lett., 5, 539-542, 1978.

Collins, R. L., A. Nomura, and C. S. Gardner, Gravity waves in the upper mesosphere over Antarctica: Lidar observations at the south pole and Syowa, J. Geophys. Res., 99, 5475-5485, 1994.

Fritts, D. C. and P. K. Rastogi, Convective and dynamical instabilities due to gravity wave motions in the lower and middle atmosphere : Theory and observations, Radio Sci., 20, 1247-1277, 1985.

Gardner, C. S. and J. D. Shelton, Density response of neutral atmospheric layers to gravity wave perturbations, J. Geophys. Res., 90, 1745-1754, 1985.

Gardner, C. S. and D. G. Voelz, Lidar studies of the nighttime sodium layer 
over Urbana, Illinois 2. gravity waves, J. Geophys. Res., 92, 4673-4694, 1987.

Gossard, E. E. and W. H. Hooke, Waves in the atmosphere, in Developments in Atmospheric Science, vol. 2, 456 pp., Elsevier Sci., New York, 1975.

Hines, C. O., Internal atmospheric gravity waves at ionospheric heights, Can. J. Phys., 38, 1441-1481, 1960.

Hostetler, C. A. and C. S. Gardner, Observations of horizontal and vertical wave number spectra of gravity wave motions in the stratosphere and mesosphere over the mid-Pacific, J. Geophys. Res., 99, 1283-1302, 1994.

Kwon, K. H., C. S. Gardner, S. K. Avery, and J. P. Avery, Correlative radar and airborne sodium lidar observations of the vertical and horizontal structure of gravity waves near the mesopause, J. Geophys. Res., 95, 13737-13748, 1990.

Nakamura, T., T. Tsuda, M. Yamamoto, S. Fukao, and S. Kato, Characteristics of gravity waves in the mesosphere observed with the middle and upper atmosphere radar 1. Momentum flux, J. Geophys. Res., 98, 8899-8910, 1993.

Nakamura, T., T. Tsuda, S. Fukao, H. Takahashi, P. P. Batista, R. A. Buriti, M. Tsutsumi, M. Ishi, K. Igarashi, H. Fukunishi, Y. Yamada, A. Nomura, T. D. Kawahara, K. Kobayashi, C. Nagasawa, M. Abo, and M. J. Taylor, Studies of the MLT region using the MU radar and simultaneous observations with $\mathrm{OH}$ spectrometer and other optical instruments, Adv. Space Res., 19, 643-652, 1997.
Namboothiri, S. P., T. Tsuda, M. Tsutsumi, T. Nakamura, C. Nagasawa, and M. Abo, Simultaneous observations of mesospheric gravity waves with the MU radar and a sodium lidar, J. Geophys. Res., 101, 4057-4062, 1996.

She, C. Y., J. R. Yu, J. W. Huang, C. Nagasawa, and C. S. Gardner, Na temperature lidar measurements of gravity wave perturbations of wind, density and temperature in the mesopause region, Geophys. Res. Lett., 18, 1329-1331, 1991.

Taylor, M. J., D. C. Fritts, and J. R. Isler, Determination of horizontal and vertical structure of an unusual pattern of short period gravity waves imaged during ALOHA-93, Geophys. Res. Lett., 22, 2837-2840, 1995.

Tsuda, T., S. Kato, T. Yokoi, T. Inoue, M. Yamamoto, T. E. VanZandt, S. Fukao, and T. Sato, Gravity waves in the mesosphere observed with the middle and upper atmosphere radar, Radio Sci., 26, 1005-1018, 1990.

Tsutsumi, M., T. Tsuda, T. Nakamura, and S. Fukao, Temperature fluctuations near the mesopause inferred from meteor observations with the middle and upper atmosphere radar, Radio Sci., 29, 599-610, 1994.

K. Kobayashi (e-mail: kkoba@jda-trdi.go.jp), T. Kitahara, T. D. Kawahara, Y. Saito, A. Nomura, T. Nakamura, T. Tsuda, M. Abo, C. Nagasawa, and M. Tsutsumi 